\title{
Fine Diffraction Effects in Si Single Crystals Implanted with Fast Ar Ions and Annealed
}

D. Żymierska ${ }^{a, *}, \mathrm{~K}$. GodWOD ${ }^{a}$, J. AdamcZeWSkA ${ }^{a}$, J. Auleytner ${ }^{a}$, J. Choiński ${ }^{b}$ And K. Regiński ${ }^{c}$

${ }^{a}$ Institute of Physics, Polish Academy of Sciences al. Lotników 32/46, 02-668 Warsaw, Poland

${ }^{b}$ Heavy Ion Laboratory of Warsaw University

Pasteura 5a, 02-093 Warsaw, Poland

${ }^{c}$ Institute of Electron Technology, al. Lotników 32/46, 02-668 Warsaw, Poland

The paper presents high-resolution X-ray diffraction studies performed for Si single crystal: as-grown, implanted with a $5 \times 10^{14}$ ions $\cdot \mathrm{cm}^{-2}$ dose of $3 \mathrm{MeV} / \mathrm{n}$ Ar ions, as well as implanted and annealed in a very high vacuum. The results are discussed on the basis of rocking curves and the mathematical analysis of the reciprocal space maps. It is shown that the lattice parameter is increased in an implanted part of the crystal, but long distance lattice curvature is not present. After annealing full relaxation of the crystal is stated.

PACS numbers: 61.80.Jh, 61.80.-x, 61.10.-i, 85.40.Ry

\section{Introduction}

The first observation of a specific damage induced by fast heavy ions in solid was made by means of transmission electron microscopy in mica irradiated by uranium fission fragments [1]. Since that many investigations were carried out in different materials by means of a number of methods [2-5], especially transmission electron microscopy (TEM) [6, 7]; also X-ray technique was successful $[8,9]$. The structural distortions induced by implantation with fast heavy ions (of energies above $1 \mathrm{MeV} / \mathrm{n}$ ) change properties of a target material. In the case of semiconductors these changes can be applied for new electronic device technology.

*corresponding author; e-mail: zymier@ifpan.edu.pl 
The aim of the present paper is X-ray diffraction study of fine structural changes introduced by implantation with fast inert gas ions, namely with $3 \mathrm{MeV} / \mathrm{n}$ Ar ions, and annealing in a silicon single crystal, which is a model semiconductor. Up to now, several X-ray studies of silicon crystals implanted with Ar and $\mathrm{Kr}$ ions were performed but for much higher energies (of the order of 1-2 GeV, i.e., $44 \mathrm{MeV} / \mathrm{n}$ ) [10-12]. In the cited investigations the following X-ray methods were applied: projection topography in Laue geometry and section topography in Bragg geometry.

Previously we investigated by means of the X-ray diffraction methods the influence of the implantation with a $1 \times 10^{14}$ ions $\cdot \mathrm{cm}^{-2}$ dose of $4 \mathrm{MeV} / \mathrm{n}$ neon ions on the silicon matrix [13], as well as the following annealing at a high temperature [14]. It was shown that implantation caused disturbance of silicon crystal structure and the increase in the lattice parameter by $1 \times 10^{-3} \mathrm{~nm}$. The present research is devoted to an effect of irradiation with the similar dose at energy of the same order ions of the next inert gas in the periodic table, namely argon.

The X-ray diffraction studies were carried out for the Si crystal: (i) as-grown (sample Gr), (ii) implanted with the fast Ar ions (sample Im), and (iii) implanted and then annealed (sample An). The results are discussed on the basis of rocking curves and the mathematical approach to analysis of reciprocal space maps described for the first time in [15].

\section{Experimental}

The dislocation-free silicon single crystal was grown by the Czochralski method. The 110 oriented $3 \mathrm{~mm}$ thick plate studied was implanted with a $5 \times 10^{14}$ ions $\cdot \mathrm{cm}^{-2}$ dose of the $3 \mathrm{MeV} / \mathrm{n}$ Ar ion beam from a $K=160$ Cyclotron at Heavy Ion Laboratory of the Warsaw University [16]. The beam current was equal to $50 \mathrm{enA}$. The implantation was performed at room temperature by a uniformly defocused beam. The mean range of the $3 \mathrm{MeV} / \mathrm{n}$ argon ions in the silicon crystal was calculated by means of a TRIM 5.3 program [17].

The annealing of the implanted specimen in a high vacuum $\left(\sim 10^{-10}\right.$ torr $)$ was divided into several steps. First, the crystals were subjected to a preannealing ( 1 hour at $473 \mathrm{~K}+1$ hour at $673 \mathrm{~K}$ ) in order to obtain low pressure in the chamber and for preliminary cleaning of the sample surface. Then, a high temperature treatment was applied. During the main annealing process the pressure also slowly increases in the chamber, so we divided the process into two parts, applying two temperature regimes. During 1 hour the temperature rose from $673 \mathrm{~K}$ to $973 \mathrm{~K}$, next for 1 hour the temperature was kept constant, and finally during 20 min the temperature was reduced up to $473 \mathrm{~K}$.

The X-ray diffraction studies were carried out by means of a Philips high-resolution MRD diffractometer equipped with a Ge 220 Bartels monochromator and a two-reflection $220 \mathrm{Ge}$ analyser. The characteristic $\mathrm{Cu} K_{\alpha_{1}}$ radiation 
$(\lambda=0.15406 \mathrm{~nm})$ was applied. The investigated samples were set up for the symmetrical 440 reflection. The rocking curves were measured with the open detector, whereas the reciprocal space maps were recorded using the analyser in front of the detector.

\section{Results}

The symmetrical 440 reflection rocking curves ( $\omega$ scan) for the as-grown silicon crystal, for the implanted with fast Ar ions specimen, and for the irradiated and next annealed sample are presented in Fig. 1 as the solid line, dotted line, and dashed line, respectively. The rocking curves for Gr sample and for An sample are very similar. The rocking curve for Im sample shows a weak diffuse peak on the left side of the main maximum indicating a formation of strains in the interior of the sample. Recently it was shown that the distortion of the rocking curve in the silicon crystal implanted with nitrogen by plasma immersion ion implantation was caused by strains in the implanted region [18]. The intensity of the main maximum of Im sample is slightly higher than maxima of other two rocking curves, that means that the Ar ion shot-through layer is not defect-free.

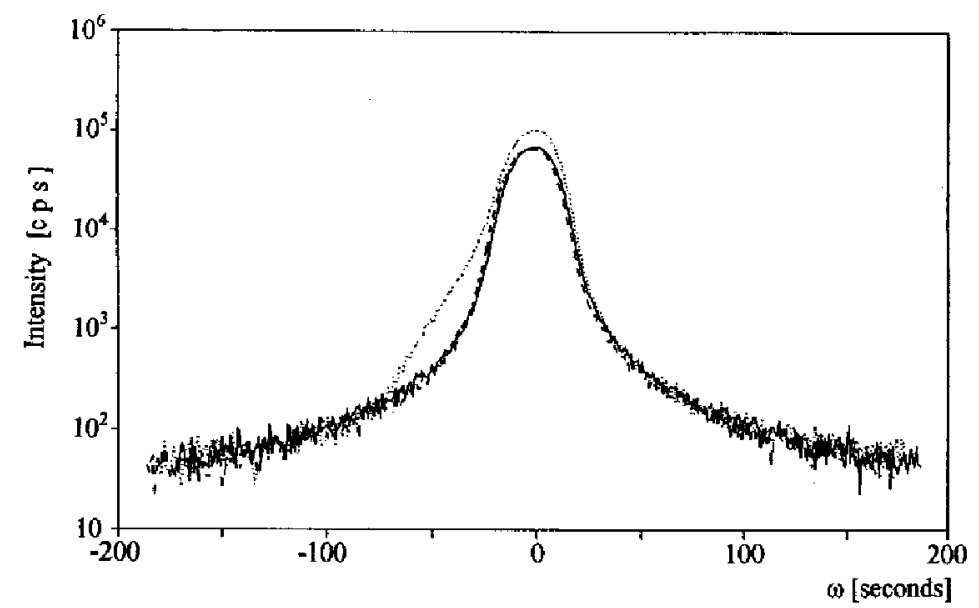

Fig. 1. The rocking curves (440 reflection) measured with the $\mathrm{Cu} K_{\alpha_{1}}$ radiation. The solid line is the rocking curve for the virgin silicon crystal, the dotted line - for the implanted with the $5 \times 10^{14}$ ions $\cdot \mathrm{cm}^{-2}$ dose of the $3 \mathrm{MeV} / \mathrm{n}$ energy argon ions sample, and the dashed line - for the irradiated and next annealed specimen.

The maps around the 440 reciprocal lattice point are given in Fig. 2. Comparing the map after the implantation (Fig. 2b) to that of the as-grown crystal (Fig. 2a), one can see that the former map is distorted. The map for An sample (Fig. 2c) is like that for Gr sample that indicates that after annealing the crystal comes back to the almost initial state. 


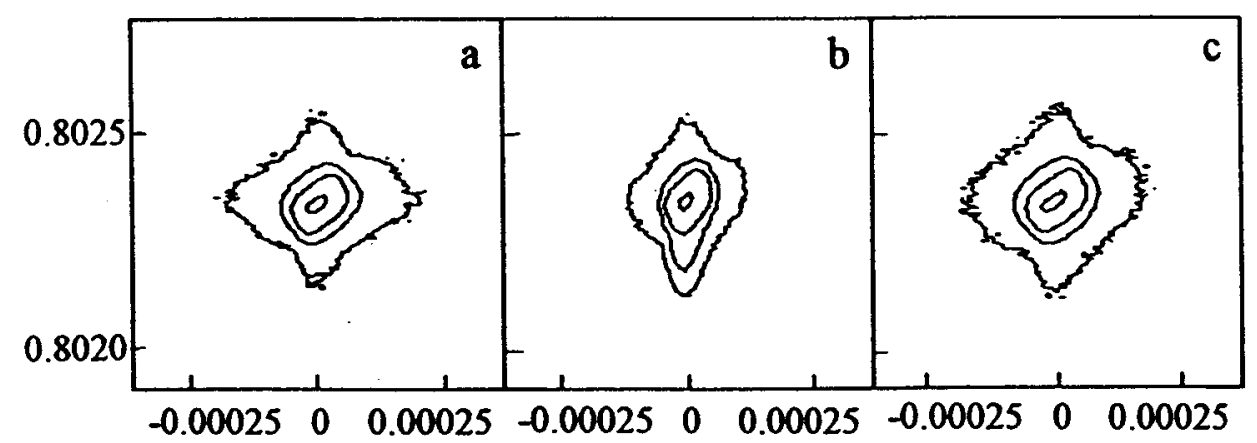

Fig. 2. The 440 reciprocal space maps for silicon crystals: (a) for the virgin sample, (b) for the irradiated crystal, and (c) for the implanted and then annealed sample. The axes are marked in units of $\lambda / 2 d$ with $\lambda$ being the wavelength of the incident radiation and $d$ interplanar distance. The neighbouring contours represent the intensity ratio equal to 10 .

\section{Analysis of the results and discussion}

The calculated mean range of the $3 \mathrm{MeV} / \mathrm{n}$ argon ions in silicon is $29.9 \mu \mathrm{m}$ that means that $\mathrm{Ar}$ ions penetrate the $\mathrm{Si}$ crystal up to about $30 \mu \mathrm{m}$. This indicates the required depth of penetration of X-rays during diffraction experiment. Next, we calculated the penetration depth for the 440 reflection of $\mathrm{Cu} K_{\alpha_{1}}$ radiation in $\mathrm{Si}$ single crystal for the Bragg case. We revealed that the main maximum stands for the reflection from the near-surface layers up to $2 \mu \mathrm{m}$, and that the penetration depth achieves $50 \mu \mathrm{m}$ for the tail ends.

The analysis of the reciprocal space map allows one to determine the microstructure of the investigated crystal and to distinguish the different effects. The assumptions and the way of performing the analysis were presented in [15]. The calculated functions: the interplanar spacing distribution function $F 1(2 \theta)$ and the orientation distribution function $F 2(\omega)$ describe lattice strains and a lattice bending, respectively. We assume that the reciprocal space map for the as-grown crystal is a reference map that means that $F 1(2 \theta)$ and $F 2(\omega)$ for the Gr crystal characterise the experiment considered, i.e., they are the instrumental functions for the high-resolution diffractometer. The results of the performed analysis are presented in Figs. 3, 4, and 5.

In Fig. 3 the runs of $F 1(2 \theta)$ functions are given for $\mathrm{Gr}$ sample (solid line in Fig. $3 \mathrm{a}$ and b), for Im plate (open circles in Fig. 3a), and An sample (open triangles in Fig. 3b). The function $F 1(2 \theta)$ for Im specimen (Fig. 3a) reveals the additional diffuse maximum. To characterise more precisely this asymmetrical broadening of $F 1(2 \theta)$ we split up the function into two component functions in the way shown in Fig. 4. The first component function corresponds to the whole sample with the same lattice parameter (open triangles), and the second component function is 

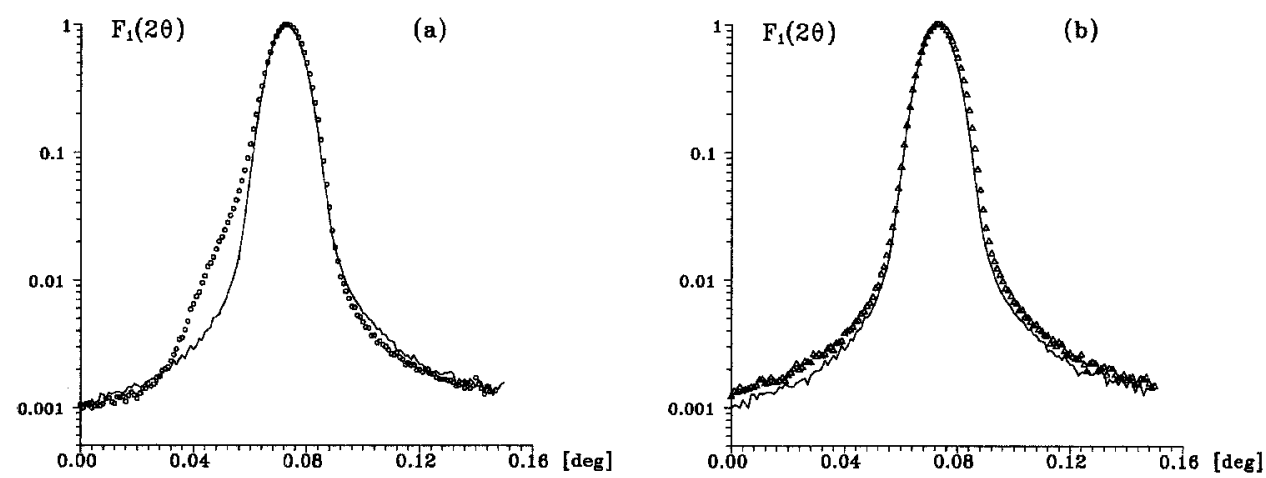

Fig. 3. The runs of $F 1(2 \theta)$ functions in a logarithmic scale obtained from the analysis of the reciprocal space maps shown in Fig. 2: (a) from the map b for the fast Ar ions implanted sample (open circles), and (b) from the map c for the irradiated and then annealed specimen (open triangles). The reference $F 1(2 \theta)$ function for the virgin Si crystal (map a) is given by a solid line.

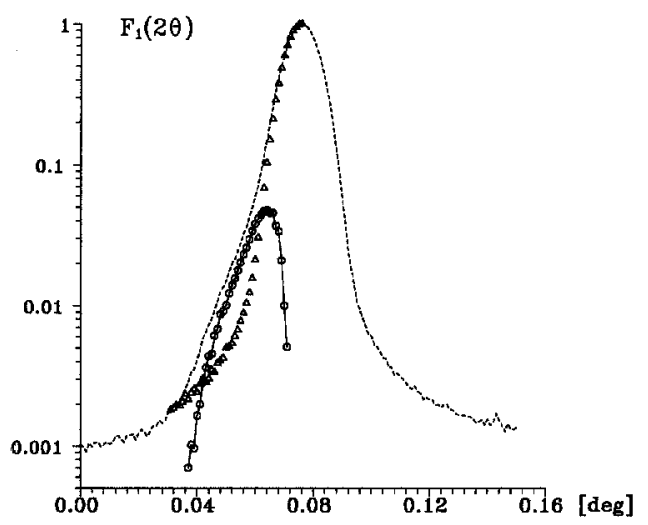

Fig. 4. The splitting of the $F 1(2 \theta)$ function for the $\mathrm{Si}$ sample implanted with fast Ar ions: the calculated $F 1(2 \theta)$ function (dashed line), the first component function corresponding to the whole sample with the same lattice parameter (open triangles), and the second component function for the part of the crystal volume with a larger lattice parameter (open circles).

connected with the part of the crystal volume of the extended lattice parameter (open circles).

From the analysis of $F 1(2 \theta)$ for Im sample it follows that the additional diffuse maximum is of 1-2 orders less intense than the main maximum. The shift of this additional peak (between $2 \theta=0.01^{\circ}$ and $0.03^{\circ}$ in the low angles direction) corresponds to the relative change of interplanar distance of $\Delta d / d=+1.3-1.5 \times 10^{-4}$, i.e., to the increase in the lattice parameter of $0.7-2.1 \times 10^{-2} \mathrm{~nm}$. The broadening 
of the function $F 1(2 \theta)$ for Im sample (open circles in Fig. 3a) of about $10-50 \%$ in comparison with the reference function (solid line in Fig. 3a) in a complete function run shows an appearance of strains perpendicular to the reflecting planes caused by the creation of extended defects. The point defects and quasi-point defects in the near-surface Ar ion shot-through layers consist in substitution in part Ar ions in Si atom rows, and they cause a lattice expansion. Argon is chemically inert, and it is why it causes only strain of crystal lattice.

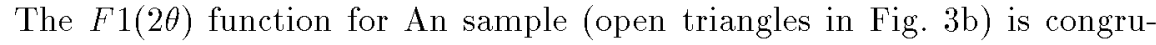
ent to the reference function (the solid line). Alike for $\mathrm{Gr}$ crystal the function is symmetrical; the additional diffuse maximum disappeared. In comparison with the $F 1(2 \theta)$ for Im sample it is broadened by about $3.4 \%$ in the half-width. Because the radiation penetration depth corresponding to the half-width does not achieve $5 \mu \mathrm{m}$, this broadening reveals formation of the extended defects within the near-surface shot-through layers. On the other hand, after annealing the $F 1(2 \theta)$ function is narrowed from $3 \%$ in the level of 0.1 of the main maximum intensity to $20 \%$ in that of 0.005 , which indicates the large relaxation of strains in the stopping power region as a result of the annealing (migration of the Ar ions to interstitial positions).
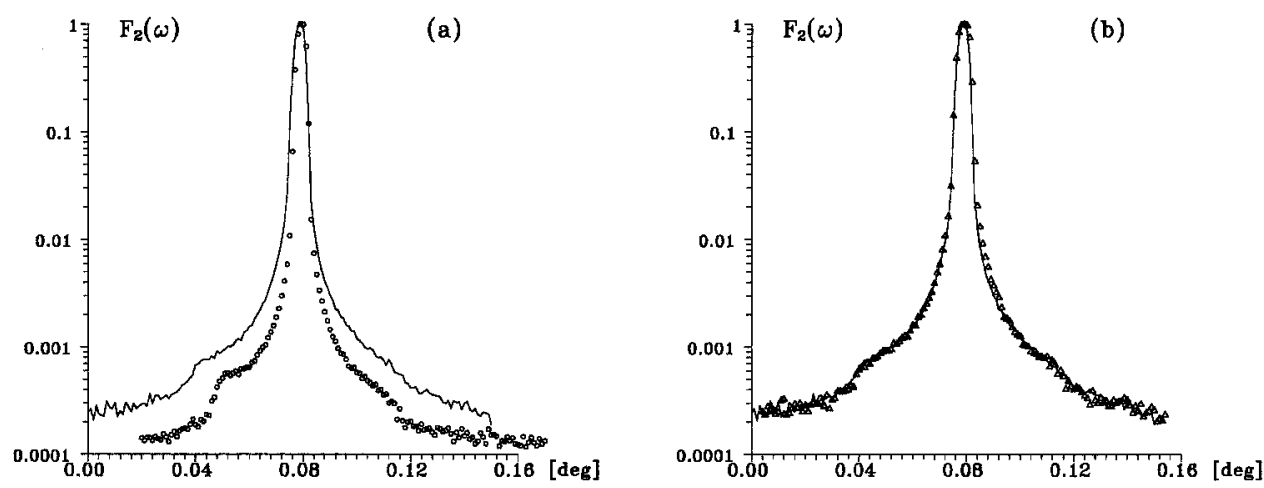

Fig. 5. The runs of $F 2(\omega)$ functions in a logarithmic scale obtained from the analysis of the reciprocal space maps given in Fig. 2: (a) open circles for Im sample (map 2b), and (b) open triangles for An sample (map 2c). The solid line presents the reference $F 2(\omega)$ function for Gr specimen (map 2a).

In Fig. 5 the runs of $F 2(\omega)$ functions for Gr sample (solid line in Fig. $5 \mathrm{a}$ and b), for Im plate (open circles in Fig. 5a), and for An sample (open triangles in Fig. $5 \mathrm{~b}$ ) are presented. In the region of the intensities from 1.0 to 0.1 the functions $F 2(\omega)$ for $\mathrm{Gr}$ and $\mathrm{Im}$ samples are almost the same, whereas in the tail region, corresponding to the X-ray penetration depth from 20 to $50 \mu \mathrm{m}$, this function for Im sample is narrowed. The calculated mean range of argon ions in silicon is about $30 \mu \mathrm{m}$ that means that in this region maximum deformation of crystal lattice 
occurs. This is the reason of lowering the diffracted intensity in the tails region in comparison with the case of Gr sample, as well as a horizontal narrowing of the reciprocal space map (Fig. $2 b$ ). The function $F 2(\omega)$ for An sample is identical as the function $F 2(\omega)$ for Gr sample (Fig. 5b) that indicates strain relaxation. More details will be presented in the next paper (in preparation).

\section{Conclusions}

The aim of the present paper was the study of fine structural changes induced by implantation with fast Ar ions into a silicon single crystal and following annealing in a high vacuum. For the research we applied an X-ray diffraction technique, namely the rocking curve measurements and the reciprocal space map imaging.

The rocking curve for the implanted sample revealed an additional peak in the left side of the main maximum. After the annealing this peak fell off and the rocking curve became very like that for the virgin crystal that means that as a result of annealing the post-implantation structural changes disappeared.

From the mathematical analysis of the reciprocal space maps it follows that:

1. The implantation with the fast Ar ions caused fine changes in the Si lattice structure. For the left tail region of the $F 1(2 \theta)$ function the additional diffuse maximum was revealed which was connected with the dispersion of the inter-planar spacings, that means the increase in the lattice parameter of about $0.7-2.1 \times 10^{-2} \mathrm{~nm}$ in the stopping power region.

2. After annealing the half-width of the $F 1(2 \theta)$ function slightly increased indicating the formation of the extended defects within the near-surface shot-through layers, whereas in the tail region the additional peak disappeared and the $F 1(2 \theta)$ function width decreased, that means a large relaxation of strains in the stopping power region.

3. The unexpected narrowing of the function $F 2(\omega)$ for the implanted crystal in the tail region can be explained by the fact that when the angular distance from the middle of the interference region rises then X-ray beam penetrates the deeper layers in the sample and achieves deformed layers which in consequence gives a decrease in the diffracted intensity.

\section{References}

[1] E.C.H. Silk, R.S. Barnes, Philos. Mag. 7, 970 (1959).

[2] R.L. Fleischer, P.B. Price, R.M. Walker, Nuclear Tracks in Solids, University of California Press, Berkeley 1975.

[3] C. Lehmann, Interaction of Radiation with Solids and Elementary Defects Production, North-Holland, Amsterdam 1977.

[4] E. Balanzat, Radiat. Effects Defects Solids 126, 97 (1993). 
[5] P. Sigmund, Nucl. Instrum. Methods, Phys. Res. B 135, 1 (1998).

[6] R. Scholz, J. Vetter, S. Hoppe, Radiat. Effects Defects Solids 126, 275 (1993).

[7] A. Dunlop, J. Henry, G. Jaskierowicz, Nucl. Instrum. Methods, Phys. Res. B 146, 222 (1998).

[8] J. Auleytner, in: Defects in Crystals, Ed. E. Mizera, World Scientific, Singapore 1988 , p. 46.

[9] J. Auleytner, J. Bąk-Misiuk, Z. Furmanik, M. Toulemonde, J. Vetter, Radiat. Effects Defects Solids 115, 335 (1991).

[10] A. Barbu, J.C. Jousset, G. Martin, M. Toulemonde, Nouvelles des GANIL 9, 16 (1985).

[11] J.N. Górecka, J. Auleytner, Phys. Status Solidi A 137, 309 (1993).

[12] J. Auleytner, Nucl. Instrum. Methods, Phys. Res. B 87, 124 (1994).

[13] J. Auleytner, D. Żymierska, J. Adamczewska, J. Domagała, J. Górecka, T. Czosnyka, L. Datsenko, V. Khrupa, Universitatis Iagellonicae Folia Physica XXXIX, 75 (1998).

[14] L.I. Datsenko, J. Auleytner, A. Misiuk, V.P. Klad'ko, V.F. Machulin, J. Bąk-Misiuk, D. Żymierska, I.V. Antonova, V.M. Melnyk, V.P. Popov, T. Czosnyka, J. Choiński, Semicond. Phys., Quantum Electronics and Optoelectronics 2, 56 (1999).

[15] D. Żymierska, J. Auleytner, K. Godwod, J. Domagała, L. Datsenko, J. Choiński, $J$ Alloys Comp. 328, 237 (2001).

[16] Heavy Ion Laboratory Annual Reports, Warsaw University, Warsaw, 1993-1999.

[17] J.P. Biersack, L.G. Haggmark, Nucl. Instrum. Methods 174, 257 (1980).

[18] E. Abramof, A.F. Beloto, M. Ueda, G.F. Gomes, L.A. Berni, H. Reuther, Nucl. Instrum. Methods, Phys. Res. B 161-163, 1054 (2000). 